\title{
Underestimated self-perception of stepping-forward decreases the risk of falls in community dwelling older adults
}

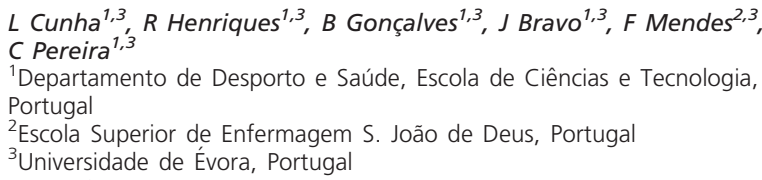

\section{Introduction:}

The impact and the high costs that falls represent for older adults, family, social, health and economic community, are a constant concern such as respect for the human imension and the fight against social exclusion. The priority of social projects and agents is to identify risk factors in order to prevent falls and to guarantee an aging without fear, without injury, without disabilities and without social dependence.

Objectives:

The present study aimed to analyse the role of the estimated and the real stepping- forward (SF) in the prevalence of falls in older adults from Alentejo region, Portugal.

Methods:

A total 113 males and 395 females aged 65 years or over were evaluated in the Stepping-forward Affordances Perception test, which evaluates the accuracy of the perceived capacity of the action limit in order to overcome a horizontal obstacle with one step. Body mass index (BMI) age, fear of falling (FES), and falling prevalence were also assessed. Associations between variables were analysed using Pearson correlation or binary regression analysis by means of SPSS software package. Statistical significance was set at $\mathrm{P} \leq .05$.

Results:

Negative correlations were observed between age, BMI, FES and real SF (r: -0.405 to $-0.183, \mathrm{p}<.01$ ) or estimated SF ( $\mathrm{r}$ : 0.141 to $-0.339, \mathrm{p}<.01)$. Increased real $\mathrm{SF}$ decreased the likelihood of falling (OR: 0.945) as well as an error tendency of underestimation (OR:0.549), while increased FES increased this likelihood (OR: 1.054), $\mathrm{p}<.05$.

Conclusions:

Older adults with poor real SF and overestimation SF bias reveal to be at high risk of falls. These results can provide a simple method for professionals and technicians identify the propensity to fall community dwelling older adults. 